Print ISSN: 2233-4165 / Online ISSN: 2233-5382

doi:http://dx.doi.org/10.13106/ijidb.2019.vol10.no7.17

\title{
Review of Corporate Social Responsibility Effects Studies
}

\author{
Seong-Jin $\mathrm{KIM}^{*}$, Sang-Cheol $\mathrm{KIM}^{* *}$
}

Received: May 21, 2019. Revised: June 19, 2019. Accepted: July 05, 2019.

\section{Abstract}

Purpose - In this study, previous CSR researches was reviewed to help understand the CSR effects, and offer suggestions for future research directions.

Research design, data, and methodology - To achieve the purpose of this study, previous CSR effects related researches were reviewed based on stakeholder perspectives.

Results - After reviewing, some limitations were found. First, most of the CSR related studies in the management area have focused on CSR effects on individual stakeholders such as the shareholder, the employee, and the consumer. Second, it was difficult to find studies on the management aspect of CSR from both an effective and efficient perspective.

Conclusions - To overcome these limitations of previous studies, several studies need to be conducted in the future. First, CSR research must be expanded into B-to-B businesses. Few studies try to figure out the effect of CSR on B-to-B businesses but only suggest the effect of CSR on B-to-B business conceptually. Therefore, empirical study needs to be conducted about CSR to show business performance in the B-to-B market. Second, the process of CSR must be developed on how to improve CSRs' effectiveness and efficiency. Finally, a relationship between a company and individual stakeholders needs to be revised using stakeholders' multiple identities.

Keyword: Coproate Social Responsibility, Stakeholder Approach, CSR Effect, CSR Definitions by Economic School.

JEL Classifications: M10, M14, M31.

\section{Introduction}

In their article, Day and Montgomery (1999) insisted "it has proven difficult to assess the net contributions of marketing to societies and economies as a whole." They further suggested that answers are needed to the following questions in order to solve the problem by theories and evidence: "Who are the stakeholders to be considered? What criteria should be used to judge the societal value? How might society seek to preserve the benefits and

* First Author, Instructor, Department of Business Administration, Seoul Cyber University, South Korea,

E-mail : socialrev@gmail.com

** Correspondent author, Professor, Majored in distribution management, department of business administration, Yuhan University, Korea. Tel: +82-2-2610-0865,

E-mail: kimsc@yuhan.ac.kr.

()) Copyright: Korean Distribution Science Association (KODISA)
This is an Open Access article distributed under the terms of the Creative Commons Attribution Non-Commercial License (https://creativecommons.org/licenses/by-nc/4.0/) which permits unrestricte non-commercial use, distribution, and reproduction in any medium, provided the original work is properly cited. minimize the negative aspects?" (Day \& Montgomery, 1999) Upon closer inspection, these answers can be found in Corporate Social Responsibility (CSR). An evolution of the CSR as a concept is related to the definition of a stakeholder whose company must consider these responsibilities.

In the early to mid 1900's, the subject concerning whose company takes the responsibility was limited to the shareholders. During the past five decades, a range of corporate responsibilities has extended to society as a whole. This change can be found in the definition of marketing. American Marketing Association (2007) defined marketing as "the activity, set of institutions, and processes for creating, communicating, delivering, and exchanging offerings that have value for customers, clients, partners, and society at large." According to the new marketing definition, marketing subjects include not only customers, clients, partners who have direct relationships to the business, but also society as a whole which is relatively disregarded. This means that marketers must be able to 
create, communicate, deliver, and exchange values for various stakeholders. CSR can play this role (Joyner \& Payne, 2002; Luo \& Bhattacharya, 2006).

The definition of CSR provides the standard for judging a social value. By Carroll's definition (1979), CSR must meet social expectations as the standards for judging constantly evolving social values. Simply stated, a social expectation to a corporation is a social value. This social value covers not only various non-profit activities for society, but also economic activities seeking profits. Finally, CSR also provides how companies can protect social benefits and minimize negative aspects of marketing. Some aspects of negative effects in marketing have occurred due to conflicts between stakeholders. Carroll (1991) claimed that CSR is probably the most useful tool in solving conflicts and balancing stakeholders' demands (Carroll, 1991).

Many studies of CSR have focused on the effects of CSR on corporate performances. In the area of management, many researchers have recognized CSR as a useful tool to manage relationships between companies and stakeholders, and they have tried to prove the effects of CSR on these afore mentioned relationships (Abbott \& Monsen, 1979; Alexander \& Buchholz, 1978; Aupperle, Carroll, \& Hatfield, 1985; Bowman \& Haire, 1975; Bragdon \& Martin, 1972; Moskowitz, 1972; Parker \& Eilbirt, 1975; Vance, 1975). However, after the initial research mentioned above, other more progressive researchers have extended their studies into the effects of CSR on employee and consumer behavior (Brown \& Dacin 1997; Folkes \& Kamins 1999; Freeman 1992; Klein \& Dawar 2004). And still, these studies about CSR effects have some limitations. First, most of the CSR related studies in the management area have focused on CSR effects on individual stakeholders such as the shareholder, the employee, and the consumer. Little study has focused on B-to-B conditions. Second, it is difficult to find some researches concerning the management of CSR both effectively and efficiently. Previous studies about CSR have dealt with the relationship between CSR and corporate performances. These studies do not address CSR management that will increase the CSR effect on corporate performance. In this study, previous CSR researches will be reviewed, and future research directions will be suggested

\section{Different perspectives of economic schools on CSR}

Until the 1960's, CSR related studies focused on the social responsibility of businessmen, not related to social responsibilities of corporations. According to Bowen's book, Christian Ethics and Economic Life (1953), he defined CSR as "the obligations of businessmen to pursue those policies, to make those decisions, or to follow those lines of action which are desirable in terms of the objectives and values of our society." Using the initial concept of businessmen's social responsibilities, Frederick (1960) stated that businessmen should oversee the operation of an economic system that fulfills the expectations of the public. According to Davis and Bloomstrom's book, Business and its Environment (1966), CSR was defined as "a person's obligation to consider the effects of his decision and actions on the whole social system. Businessmen apply social responsibility when they consider the needs and interest of others who may be accepted by business actions." These definitions of corporate responsibility of businessmen were accepted as the main concepts in the middle of the 1960's (Heald, 1970).

However, many companies changed to "corporations" during the 1970's, and an owner was not considered responsible for making total decisions. The CEO who was empowered by shareholders to make decisions for the corporations had some problems with profit sharing. Shareholders asserted their rights of overall corporate profits, whereas certain societies insisted that a part of corporate profits that were created in a society should be used for social welfare. Moreover, many problems such as labor, exhaustion of natural resources, or environmental pollution, which occurred through corporate growth, were acknowledged by many people and groups as being the results of selfish behaviors of corporations and demanded responsible activities of corporations for solving these problems.

Who is CSR responsible to? This question has been widely discussed for many decades. Many economists have tried to answer this question. Classic economists had approached CSR with a cost perspective. According to the classic economists' view, a basic goal of corporations was to maximize shareholder's profits, and corporations had responsibilities only to shareholders. For increasing profits, corporations should decrease their costs. But, CSR increased unnecessary costs. Therefore, corporations should not invest their resources to CSR in order to protect a shareholder's interest. In the 1970's, neo-classic economist criticized the classic economics' view, because classic economist focused on a short-term financial performance. According to the neo-classic economics' view, they accepted a basic idea of classic economics: CSR increased costs and decreased profits into short term. But they had different views on the long-term effects of CSR. Though they agreed with the classic economists' view of a range of CSR, neo-economists insisted that CSR would be able to increase profits in the long term.

Social economists claimed that the range of corporate responsibility should be extended to the whole society. Based on the relationship between corporations and stakeholders, corporations had responsibilities not only to shareholders, but also to all the stakeholders. Therefore, they insisted that corporations had to use their resources and profits to improve the social welfare. Additionally, they 
had tried to prove the relationship between CSR and the short-term performance of corporations. Beginning in 2000, the range of CSR was widened in its extension by ecological economists. According to the ecological economists' view, CSR should include a responsibility toward future generations. [Table 2-1] shows brief differentiations of CSR perspectives between various economic schools of thought.

Table 1: Different perspectives of CSR by economic schools

\begin{tabular}{|c|c|c|c|c|}
\hline Schools & $\begin{array}{c}\text { Classic } \\
\text { Economics }\end{array}$ & $\begin{array}{l}\text { Neo-classic } \\
\text { Economics }\end{array}$ & $\begin{array}{c}\text { Social } \\
\text { Economics }\end{array}$ & $\begin{array}{l}\text { Ecological } \\
\text { Economics }\end{array}$ \\
\hline Subject of CSR & Shareholder & Stockholder & Stakeholder & $\begin{array}{c}\text { Stakeholder } \\
\text { including } \\
\text { future } \\
\text { generations }\end{array}$ \\
\hline $\begin{array}{c}\text { Core } \\
\text { Concept }\end{array}$ & $\begin{array}{c}\text { Shareholder's } \\
\text { profit } \\
\text { maximization }\end{array}$ & $\begin{array}{l}\text { Enlightened } \\
\text { self-interest }\end{array}$ & $\begin{array}{c}\text { Stakeholder } \\
\text { management }\end{array}$ & $\begin{array}{c}\text { Sustainable } \\
\text { development }\end{array}$ \\
\hline $\begin{array}{c}\text { The relationship } \\
\text { between CSR } \\
\text { and financial } \\
\text { performance }\end{array}$ & Exclusive & Inclusive & Integrated & Integrated \\
\hline Main value & $\begin{array}{l}\text { Short-term } \\
\text { Economic } \\
\text { value }\end{array}$ & $\begin{array}{l}\text { Long-term } \\
\text { economic } \\
\text { value }\end{array}$ & $\begin{array}{c}\text { Economic } \\
\text { value }+ \\
\text { Social value }\end{array}$ & $\begin{array}{c}\text { Economic } \\
\text { value }+ \\
\text { Social value }\end{array}$ \\
\hline
\end{tabular}

\section{The effects of CSR based on the stakeholder approach}

A range of CSR is spreading out from shareholders to stakeholders and future generation with the course of time. Additionally, all companies accept CSR as a part of corporate activities. As a result, CSR-related investments are on the rise every year. These trends lead to increase an interest in the effects of CSR on a corporate management process. Many researchers in management area have tried to prove the effects of CSR on corporate performances based on the stakeholder approach. Therefore, previous studies about CSR are also arranged based on a specified stakeholder type in this study.

Clarkson (1995) classified stakeholders as primary and secondary stakeholder groups. Primary stakeholder groups are a complex set of relationships between and among interest groups with different rights, objectives, expectations, and responsibilities, whereas secondary stakeholders are groups who influence or affect, or are influenced or affected by, the corporation, but they are not engaged in transactions with the corporation and are not essential for its survival. Clarkson (1995) emphasized the importance of primary stakeholder groups, because "the corporation's survival and continuing success depend upon the ability of its managers to create sufficient wealth, value, or satisfaction for primary stakeholder groups." If corporations fail to retain the participation of a primary stakeholder group, its performances are worse and worse. Additionally, Donaldson and Preston (1995) classified primary stakeholder groups as the input side of stakeholders such as investors, employees, and suppliers, and the output side of stakeholders such as customers. This study also focuses on the primary stakeholder groups such as investors, employees, suppliers, and customers following Clarkson's classification (1995).

\subsection{CSR researches toward investors}

Earlier studies about CSR effects had focused on the relationship between CSR and corporate financial performances, because it was necessary to prove the positive relationship between CSR and corporate financial performance in order to justify activities related to CSR. The results of those studies are complicated. The following [Table 2-4] shows briefly the results of CSR related studies toward investors in a management area.

Many researchers have tried to find the positive relationship between CSR and corporate financial performance using ROI, ROE, or other financial indicators, and some researchers succeeded in proving the relationship (Freeman, 1997; Griffin \& Mahon, 1997; Kang, Germann, \& Greewa, 2016; Key \& Popkin, 1998; Kiessling, Isaksson, \& Yasar, 2016; Roman, Hayibor, \& Agle, 1999; Waddock \& Graves, 1997). Additionally, it was found that CSR has a positive influence in a stock price (Alexander \& Buchholz, 1978). Hillman and Keim (2001) also proved that building better relations with primary stakeholders could lead to increased shareholder wealth by increasing competitive advantage. On the other side, Bowman and Haire (1975) insisted a relation between CSR level and ROE is a 'U-shaped curve.' It means that companies who have extremely high or low CSR make better profits than middle level companies.

As mentioned above, earlier CSR related studies mainly have tried to verify the positive effects of CSR on the relationship with investors or shareholders indirectly by proving the relationship between CSR and corporate financial performances. But some researchers have tried to verify directly the effects of CSR on the behaviors or intentions of investors or shareholders. Ullmann (1985) showed that increasing the evaluations about CSR activities decreased the investors' informational uncertainty toward the company. Sen, Bhattacharya, and Korschun (2006) also proved that awareness of a company's CSR has a positive influence on intention to invest. Through these results, it is able to confirm the positive effects of CSR on the relationship with investors. 
Table 2: CSR researches related to investor relations

\begin{tabular}{|c|c|c|c|}
\hline Researcher & Antecedent & Mediator/Moderator & Consequence \\
\hline Abbott \& Monsen (1979) & - CSR disclosures & - Number of employees & - ROI \\
\hline $\begin{array}{l}\text { Alexander \& Buchholz } \\
\text { (1978) }\end{array}$ & - CSR & & - Stock market performance \\
\hline Aupperle et al. (1985) & - Orientation toward CSR & & - Profitability (ROA) \\
\hline Bowman \& Haire (1975) & $\begin{array}{ll}\text { - } & \text { Discussion of CR } \\
\text { - } & \text { Mention of CR } \\
\text { - } & \text { CEP pollution index } \\
\text { - } & \text { Outside nonbusiness director }\end{array}$ & $\begin{array}{l}\text { - Consumer vs. nonconsumer } \\
\text { company }\end{array}$ & $\begin{array}{l}\text { Profitability (mean ROE, } \\
\text { median ROE) }\end{array}$ \\
\hline $\begin{array}{l}\text { Cochran and Wood } \\
\text { (1984) }\end{array}$ & - CSR & & $\begin{array}{l}\text { - Financial performance } \\
\text { - Asset age } \\
\text { - Asset turnover }\end{array}$ \\
\hline $\begin{array}{l}\text { Hamilton, Jo, and Statman } \\
\text { (1993) }\end{array}$ & $\begin{array}{l}\text { Type of portfolio (socially } \\
\text { responsible portfolio, } \\
\text { conventional portfolio) }\end{array}$ & & - Expected return of portfolio \\
\hline Hillman and Keim (2001) & $\begin{array}{l}\text { - Stakeholder management } \\
\text { - Social issues participation }\end{array}$ & & $\begin{array}{l}\text { - Shareholder value creation } \\
\text { (market value-added) }\end{array}$ \\
\hline $\begin{array}{l}\text { Johnson and Greening } \\
\text { (1999) }\end{array}$ & $\begin{array}{l}\text { - Institutional investor types } \\
\text { - Governance devices }\end{array}$ & $\begin{array}{l}\text { - Accounting performance } \\
\text { - Firm size }\end{array}$ & $\begin{array}{l}\text { - Corporate social } \\
\text { performance }\end{array}$ \\
\hline $\begin{array}{l}\text { Sen, Bhattacharya, and } \\
\text { Korschun (2006) }\end{array}$ & $\begin{array}{l}\text { - Awareness of a company's } \\
\text { CSR }\end{array}$ & $\begin{array}{l}\text { - Stakeholder's genuine } \\
\text { concern attributions }\end{array}$ & $\begin{array}{l}\text { - Stakeholder associations } \\
\text { - Stakeholder attitude } \\
\text { - Stakeholder identification } \\
\text { - } \text { Intention to seek } \\
\text { employment } \\
\text { - Intention to invest }\end{array}$ \\
\hline Ullmann (1985) & $\begin{array}{l}\text { - Quantity and quality of a } \\
\text { firm's social disclosure }\end{array}$ & - Social performance & $\begin{array}{l}\text { - Economic performance } \\
\text { - Investors' informational } \\
\text { uncertainty }\end{array}$ \\
\hline
\end{tabular}

\subsection{CSR researches toward consumers}

Marketing academicians have focused on the effects of CSR on consumers. These researches have had an interest in intangible assets such as consumers' attitudes or behaviors, corporate or product image, WOM or purchase intention, or customer loyalty. The following [Table 2-5] shows briefly previous CSR research in the marketing area.

Some variables such as demographic variables, socio-economic variables, fitness, motivation, timing, intimacy, expectations, and related information are revealed as antecedents of consumers' CSR evaluations (Anderson Jr. \& Cunningham, 1972; Becker-Olsen, Cudmore, \& Hill, 2006; Creyer \& Ross, 1997; David, Kline, \& Yang, 2005; Deng, \& Xu, 2017; Du, Bhattacharya, \& Sen, 2007; Fatima, Rahman, \& Khan, 2015; Guzman \& Davis, 2017; Habel, Schons, Alavi, \& Wieseke, 2016; Hur, Kim, \& Woo, 2014; Idowu, vertigans, \& Burlea Schiopoiu, 2017; Karna, Hansen, \& Juslin, 2003; Kim, Hur, \& Yeo, 2015; Lee, Chang, \& Lee, 2017; Luchs \& Kumar, 2017; Maignan \& Ferrell, 2003; Marin, Cuestas, \& Roman, 2016; Miller \& Merrilees, 2013; Narwal \& Singh, 2013; Newman, gorlin, \& Dhar, 2014; Oberseder, Schlegelmilch, Murphy, \& Gruber, 2014; Perez \&
Del Bosque, 2015a, b; Sen \& Bhattacharya, 2001; Sen, Gurhan-Canli, \& Morwitz, 2001; Simmon \& Becker-Olsen, 2006; Tingchi, Liu, Wong, Shi, Chu, \& Brock, 2014). Anderson Jr. and Cunningham (1972) found that demographic and socio-economic variables have effects on consumers' social consciousness. Becker-Olsen, Cudmore, and Hill (2006) revealed independent variables of consumers' attitudes and behaviors such as fit, motivation (profit-motivated vs. socially motivated), and timing of CSR. Creyer and Ross (1997) found the relationship between consumers' expectation about ethical behavior and willingness to reward ethical behavior and to punish unethical behavior. According to researches of Follows and Jobber (2000), Kinnear, Taylor, and Ahmed (1974), Lichtenstein, Drumwright, and Braig (2004), and Sen, Bhattacharya, and Korschun (2006), perception about CSR is an important variable on CSR evaluation. It is meaningless if consumers don't recognize CSR activities, even though companies do CSR activities very well.

Many of CSR related studies have focused on consumer behavior variables as dependent variables of CSR activities (Anselmsson \& Johnson, 2006; Becker-Olsen, Cudmore, \& Hill, 2006; Carrigan \& Atalla, 2001; David, Kline, \& Yang, 
2005; Follows \& Jobber, 2000; Karna, Hansen, \& Juslin, 2003; Klein \& Dawar, 2004; Sen \& Battacharya, 2001; Sen, Bhattacharya, \& Korschun, 2006). According to all this research, some researchers proved that CSR related activities have direct effects on consumers' buying related behaviors. Other researchers found that the relationship between CSR activities and consumer's purchasing behaviors is mediated by corporate identity, consumer-corporate congruence, or behavioral control. Additionally, CSR is positively related to attitude change (Ahluwalia, Burnkrand, \& Unnava, 2000; Becker-Olsen, Cudmore, \& Hill, 2006), corporate or product evaluation (Becker-Olsen, Cudmore, \& Hill, 2006; Brown \& Dacin, 1997; Karna, Hansen, \& Juslin, 2003; Sen \& Bhattacharya, 2001), support to corporations (Handelman \& Arnold, 1999), and customer satisfaction (Luo \& Bhattacharya, 2006; Thomas, Vitell, gilbert, \& Rose, 2002).

\subsection{CSR researches related to employees}

Few studies have been conducted related to the effect of CSR on employees. The following [Table 2-6] briefly shows previous research about relationship between CSR and employees. This research has focused on employees' job performances such as role conflict, organizational commitment, job satisfaction, and turnover intention (Brammer, He, \& Mellahi, 2015; Celma, Martinez-garcia, \& Coenders, 2014; Deroeck, Arkemi, \& Swaen, 2016; De Roeck, Marizue, Stinglhamber, \& Swaen, 2014; Du,
Bhattacharya, \& Sen, 2015; Farooq, Farooq, \& Jasimuddin, 2014; Farooq, Rupp, \& Farooq, 2017; Gatignon_Tumau, \& Mignonac, 2015; Gond, El Akremi, Swaen, \& Babu, 2017; Luo \& Du, 2015; Morgeson, Aguinis, Waldman, \& Siegel, 2013; Naseer, Raja, Syed, Donai, \& Darr, 2016; Rupp, Shao, Thornton, \& Skarlicki, 2013). Employees frequently are faced with conflict situations when the relationship between a corporate profit and ethics is a tradeoff. At this time, CSR solves employees' internal conflict which is caused by a given role (Ho, Barnes, \& Desborde, 1997; Schwepker, Ferrell, \& Ingram, 1997). Furthermore, CSR makes employees commit their roles or their jobs (Maignan \& Ferrell, 2001; Maignan, Ferrell, \& Hult, 1999; Perterson, 2004). Aguilera, Rupp, Williams, and Ganapathi (2007) insisted that CSR plays an important role to justify corporate fairness, and fairness which is felt by employees has an influence on job satisfaction, commitment, turnover, or job performance. But their insistence needs to be proven practical. Additionally, CSR help corporations recruit good employees. Sen, Battacharya, and Korschun (2006) proved the positive relationship between CSR awareness and intention of employment. This effect is also identified in Porter and Kramer's study (2002). CISCO network established CISCO Network Academy which changed dramatically a local community. In turn, the local community helped CISCO increase their competitive advantage by supplying excellent human resources educated through the CISCO Network Academy.

Table 3: CSR researches related to consumers

\begin{tabular}{|c|c|c|c|}
\hline Researcher & Antecedent & Mediator/Moderator & Consequence \\
\hline $\begin{array}{l}\text { Becker-Olsen, Cudmore, } \\
\text { and Hill (2006) }\end{array}$ & $\begin{array}{l}\text { - } \text { Fit } \\
\text { - Motivation (profit-motivated, } \\
\text { socially motivated) } \\
\text { - Timing }\end{array}$ & & $\begin{array}{l}\text { - Number of thoughts } \\
\text { - Favorability of thoughts } \\
\text { - Thoughts related to } \\
\text { motive } \\
\text { - Overall attitude } \\
\text { - Purchase intention } \\
\text { - Firm credibility } \\
\text { - Firm position } \\
\text { - Firm ability }\end{array}$ \\
\hline $\begin{array}{l}\text { David, Kline, and Yang } \\
(2005)\end{array}$ & $\begin{array}{l}\text { - CSR familiarity } \\
\text { - CSR actions (relational, moral, } \\
\text { discretionary) } \\
\text { - Brand familiarity }\end{array}$ & - Corporate identity & - Purchase intention \\
\hline $\begin{array}{l}\text { Du, Bhattacharya, and } \\
\text { Sen (2007) }\end{array}$ & - CSR awareness & $\begin{array}{l}\text { - Corporate ability beliefs } \\
\text { - CSR beliefs } \\
\text { - CSR attribution }\end{array}$ & $\begin{array}{l}\text { - Company-consumer } \\
\text { identification } \\
\text { - Loyalty } \\
\text { - } \text { Advocacy }\end{array}$ \\
\hline $\begin{array}{l}\text { Follows and Jobber } \\
(2000)\end{array}$ & $\begin{array}{l}\text { - Perceived environmental } \\
\text { consequences } \\
\text { - Perceived individual } \\
\text { consequences of purchasing } \\
\text { environmental responsible } \\
\text { products }\end{array}$ & & $\begin{array}{l}\text { - environmental responsible } \\
\text { purchase intentions }\end{array}$ \\
\hline
\end{tabular}




\begin{tabular}{|c|c|c|c|}
\hline $\begin{array}{l}\text { Karna, Hansen, and } \\
\text { Juslin } \\
\text { (2003) }\end{array}$ & $\begin{array}{l}\text { - } \quad \text { CSR information } \\
\text { - } \quad \text { New product quality information }\end{array}$ & & $\begin{array}{l}\text { - Company evaluation } \\
\text { among consumers } \\
\text { - Purchase intention }\end{array}$ \\
\hline Klein and Dawar (2004) & - Product crisis & $\begin{array}{l}\text { - } \text { Blame } \\
\text { - } \text { Brand evaluation } \\
\text { - } \quad \text { CSR }\end{array}$ & - Buying intention \\
\hline $\begin{array}{l}\text { Lichtenstein, Drumwright, } \\
\text { and Braig } \\
(2004)\end{array}$ & $\begin{array}{l}\text { - Perceptions of CSR } \\
\text { - Connectedness of nonprofit } \\
\text { domain to CSR domain } \\
\text { - Company exceeding its } \\
\text { boundaries } \\
\text { - } \text { Frugality } \\
\text { - Nonprofit domain importance }\end{array}$ & $\begin{array}{l}\text { - Negative attributions about } \\
\text { the company } \\
\text { - Perceived opportunity to } \\
\text { do good } \\
\text { - Company-consumer } \\
\text { identification } \\
\text { - Perceptual corporate } \\
\text { benefits }\end{array}$ & $\begin{array}{l}\text { - Nonprofit donation } \\
\text { - Behavioral corporate } \\
\text { benefits }\end{array}$ \\
\hline $\begin{array}{l}\text { Luo and Bhattacharya } \\
(2006)\end{array}$ & $\begin{array}{l}\text { - } \text { CSR } \\
\text { - } \text { Corporate ability }\end{array}$ & - Customer satisfaction & - Market value \\
\hline $\begin{array}{l}\text { Maignan and Ferrell } \\
(2003)\end{array}$ & - Country & & $\begin{array}{l}\text { - Consumers' evaluation of } \\
\text { CSR } \\
\text { - Consumers' evaluation of } \\
\text { stakeholder responsibilities }\end{array}$ \\
\hline $\begin{array}{l}\text { Sen and Bhattacharya } \\
\text { (2001) }\end{array}$ & $\begin{array}{l}\text { - } \quad \text { CSR information } \\
\text { - New product quality }\end{array}$ & $\begin{array}{l}\text { - Company-consumer } \\
\text { congruence } \\
\text { - CSR support } \\
\text { - CSR domain } \\
\text { - CSR-corporate ability } \\
\text { beliefs }\end{array}$ & $\begin{array}{l}\text { - Company evaluation } \\
\text { - Purchase intention }\end{array}$ \\
\hline $\begin{array}{l}\text { Sen, Bhattacharya, and } \\
\text { Korschun (2006) }\end{array}$ & - Awareness of company's CSR & $\begin{array}{l}\text { - Stakeholders' genuine } \\
\text { concern attributions }\end{array}$ & $\begin{array}{l}\text { - Stakeholder associations } \\
\text { - Stakeholder attitude } \\
\text { - Stakeholder identification } \\
\text { - Purchase intention } \\
\text { - Intention to seek } \\
\text { employment } \\
\text { - Intention to invest }\end{array}$ \\
\hline Shaw and Shiu (2003) & $\begin{array}{l}\text { - External control } \\
\text { - Subjective norm } \\
\text { - Internal ethics }\end{array}$ & $\begin{array}{l}\text { - Behavioral control } \\
\text { - Internal reflection }\end{array}$ & - Behavioral intention \\
\hline $\begin{array}{l}\text { Simmons and } \\
\text { Becker-Olsen } \\
(2006)\end{array}$ & - Fit & $\begin{array}{l}\text { - Clarity of positioning } \\
\text { - Attitude toward the } \\
\text { sponsorship }\end{array}$ & - Firm equity \\
\hline
\end{tabular}

Table 4: CSR researches related to Employee

\begin{tabular}{|c|c|c|c|}
\hline Researcher & Antecedent & Mediator/Moderator & Consequence \\
\hline $\begin{array}{l}\text { Ho, Barnes, and Desborde } \\
\text { (1997) }\end{array}$ & $\begin{array}{ll}\text { - } & \text { Age, Education } \\
\text { - } & \text { Idealism } \\
\text { - } & \text { Relativism } \\
\text { - } & \text { Machiavellianism }\end{array}$ & - Cognitive moral development & $\begin{array}{ll}\text { - Role conflict } \\
\text { - Role ambiguity }\end{array}$ \\
\hline $\begin{array}{l}\text { Karande, Rao, and } \\
\text { Sihghapakdi } \\
(2002)\end{array}$ & $\begin{array}{l}\text { - } \text { Country differences } \\
\text { - Age, Gender } \\
\text { - } \text { Corporate ethical values }\end{array}$ & $\dot{\bullet}$ & $\begin{array}{ll}\text { - Idealism } \\
\text { - } & \text { Relativism }\end{array}$ \\
\hline $\begin{array}{l}\text { Mackenzie, Podsakoff, } \\
\text { Fetter } \\
(1993)\end{array}$ & $\begin{array}{l}\text { - Objective sales performance } \\
\text { - Organizational citizenship behavior } \\
\text { - conscientiousness }\end{array}$ & & $\begin{array}{l}\text { Manager's overall } \\
\text { evaluation of sales } \\
\text { personnel }\end{array}$ \\
\hline $\begin{array}{l}\text { Maignan and Ferrell } \\
\text { (2001) }\end{array}$ & $\begin{array}{l}\text { - Market orientation } \\
\text { - } \text { Humanistic orientation } \\
\text { - } \text { Competitive orientation }\end{array}$ & - Corporate citizenship & $\begin{array}{l}\text { - Employee commitment } \\
\text { - Customer loyalty } \\
\text { - Business performance }\end{array}$ \\
\hline
\end{tabular}




\begin{tabular}{|c|c|c|c|}
\hline $\begin{array}{l}\text { Maignan, Ferrell, and Hult } \\
\text { (1999) }\end{array}$ & $\begin{array}{l}\text { - Market orientation } \\
\text { - } \text { Humanistic orientation } \\
\text { - Competitive orientation }\end{array}$ & $\begin{array}{l}\text { - } \text { Corporate citizenship } \\
\text { - Employee commitment } \\
\text { - } \text { Customer loyalty }\end{array}$ & - Business performance \\
\hline $\begin{array}{l}\text { Peterson } \\
(2004)\end{array}$ & $\begin{array}{l}\text { - Employees' perceptions of the } \\
\text { firm's corporate citizenship } \\
\text { - Citizenship }\end{array}$ & $\begin{array}{l}\text { Employee's beliefs supporting } \\
\text { the importance of social } \\
\text { responsibility of businesses } \\
\text { increase } \\
\text { - Gender, age, Tenure } \\
\text { - Firm size }\end{array}$ & $\begin{array}{l}\text { - Organizational } \\
\text { commitment }\end{array}$ \\
\hline $\begin{array}{l}\text { Schwepker. Ferrell, and } \\
\text { Ingram (1997) }\end{array}$ & - Organizational ethical climate & - Ethical conflict & - Role conflict \\
\hline $\begin{array}{l}\text { Sparks and Hunt } \\
(1998)\end{array}$ & $\begin{array}{l}\text { - } \text { Organizational socialization } \\
\text { - Professional socialization } \\
\text { - Empathy } \\
\text { - Relativism } \\
\text { - Ethical training }\end{array}$ & & - Ethical sensitivity \\
\hline $\begin{array}{l}\text { Turban and Greening } \\
\text { (1997) }\end{array}$ & $\begin{array}{l}\text { Corporate social performance } \\
\text { rating }\end{array}$ & $\begin{array}{l}\text { - Organization reputation } \\
\text { - Organization size } \\
\text { - } \quad \text { Firm profitability }\end{array}$ & $\begin{array}{l}\text { - Organizational attractive } \\
\text { as employer }\end{array}$ \\
\hline
\end{tabular}

\subsection{The CSR effect on society}

Most of CSR research has focused on the relationship with individual stakeholders. But a few researchers have begun viewing CSR as a tool for a competitive advantage by solving social problems (Hart, 1995; Hart \& Christensen, 2002; Lizt, 1996; Porter \& Kramer, 2002; Prahalad, 2003; Prahalad \& Hammond, 2002; Van Rekom, Berens, \& Van Halderen, 2013). According to several researches, CSR give a company a competitive advantage by improving market conditions.

Porter and Kramer (2002) are representative research who persist CSR is a good way to improve corporate competitive advantage. They showed the proof of their persistence using the case of CISCO Network. Mentioned earlier in a study, CISCO Network, a global network device company, established CISCO Network Academy. CISCO Network Academy has provided free education to youths in the local community since it was established. As a result of this program, the level and quality of education in that local community was greatly extended. An out-growth of the new CISCO Network Academy surprisingly became an improved market for CISCO Network, as well as increased profits, and reduced employment cost.

Porter and Krammer (2002) tried to prove the effect of CSR on an improvement of an existing market, whereas Prahalad (2002) claimed a possibility of developing a new market through CSR. Traditionally, most corporate strategies have focused on middle and upper classes as main target markets. However, the population of the lower class is larger than the total sum of the population in middle and upper classes. Therefore, the lower-class population can be a great market for any companies. Prahalad (2002) insisted that it is possible to select an economic lower class as a target market. To do this, disruptive innovation is a necessary condition. Christensen, Craig, and Hart (2001) explained that disruptive innovation as a group or company identifying needs and wants of the lower-class population and developing low price products and services which meet their needs and wants. Disruptive innovation not only improves the social economic environment of the lower class in the economic pyramid, but also improves corporate competitive advantage through creating a new market.

\section{Conclusion}

\subsection{Summary}

There are many CSR related researches in the management area. This study reviewed previous CSR reaearches based on stakeholder approach.

Earlier studies about CSR effects had focused on the relationship between CSR and corporate financial performances by financial researchers, because it was necessary to prove the positive relationship between CSR and corporate financial performance in order to justify activities related to CSR. CSR related studies have verified the positive effects of CSR on the relationship with investors or shareholders directly and indirectly. Marketing researchers have focused on the effects of CSR on consumers. These researches have had an interest in intangible assets such as consumers' attitudes or behaviors, corporate or product image, WOM/purchase intention, or customer loyalty. Most of relations between CSR and marketing constructs are proved. Few studies in human resource management area have been conducted related to the effect of CSR on employees. 
The studies found CSR has an impact of on as role conflict, organizational commitmen, job satisfaction, commitment, turnover intention, and job performance. a few researchers have begun viewing CSR as a tool for a competitive advantage by solving social problems. According to several researches, CSR give a company a competitive advantage by improving market conditions in various ways.

\subsection{Implications and Suggestions for Future Research}

\subsubsection{Implications}

This study reviewed previous CSR studies based on stakeholder perspective. Preious studies are categorized under the type of stakeholder such as investors, consumers, employees, and society. This study helps to increase understanding previous CSR studies better. Someone who want to do a CSR related study can be helped understandind what kind of effects are existed in management area by this study.

After reviewing CSR related studies in management area, several questions can be found. Why is it difficult to find CSR research focusing on business to business market? Why are CSR process-related studies limited compared with CSR effect-related studies? As previous researchers said, is it possible to divide individual stakeholders clearly into a consumer group, an employee group, or an investor group?

\subsubsection{Expand CSR research area into B-to-B business}

As a result of classifying CSR researches based on stakeholder perspective, there is no study about the effect of CSR in the business to business market. The reason why a CSR study in the business to business market is not found can be inferred from the characteristic of B-to-B business. The relationship with a corporate customer is formed based on profit-seeking behavior. CSR is considered an unsuitable research topic in the business to business market. However, it is possible that CSR has an influence on B-to-B business, because CSR is intended for unspecified individuals. For example, a corporation which conducts CSR well is recognized as a law-abiding and ethical company. Then, corporate customers or partners will judge that a corporation never displays opportunistic behavior using CSR-related information. As a result of the judgment, a corporate customer can reduce unnecessary costs such as monitoring cost, or a transaction-specific investment cost. Additionally, it is possible to improve a relational performance by sharing information, increasing dependency, or increasing relationship commitment. Therefore, additional study is needed about how CSR can improve business performance in a business to business market.

\subsubsection{Developing CSR Management Framework}

CSR-related studies in management have focused on the
CSR effects. In order to help companies, it also needs to study about how to conduct CSR well. Watrick and Cochran (1985) and Wood (1991) proposed a CSR management framework composed of three stages. But Wood (1991) criticized the CSR principle in Watrick and Cochran's model as not being a principle, but a motivation of behavior, and the CSR program or policy in their model was insufficient to include all of the results of CSR activities. Additionally, Watrick and Cochran's model was closer to a stage of CSR preparation, than a whole CSR process.

Wood's (1991) model also had several limitations. First of all, three types of CSR processes in Wood's CSR framework are just a different name of CSR. Actually, issue management is used as the same meaning with CSR. Furthermore, outcome of corporate behavior such as social programs or social policy is not a result of the CSR process, but a former stage of the CSR process. Therefore, there is a limit to using both Watrick and Cochran's (1985) and Wood's (1991) models as a CSR management tool. To overcoming these limitations, New CSR process measurement has to be developed in order to manage CSR effectively and efficiently.

\subsubsection{Revise a relationship between a company and individual stakeholders}

Another limitation in previous researches classified clearly individual stakeholders into several groups. Clarkson (1995) and Donaldson and Preston (1995) suggested that stakeholders can be divided into shareholder, employee, consumer, government, or society. Previous research has been executed based on this classification. However, it is almost impossible to classify stakeholder clearly. Most stakeholders have multiple identities. Some employees hold their company stocks and purchase their company products. It means that those employees are shareholders and consumers. In this case, employees are able to feel conflict toward CSR activities. As an employee or a shareholder, they want a company to raise the price of products or services in order to increase their salary or stock dividends. But as a consumer, they want a company to lower the price in order to decrease the cost of living. In this regard, individual stakeholders have multiple relationships with a company, and come into conflict between multiple identities. Therefore, a variety of identities have to be considered in the CSR research toward individual stakeholders.

Many companies use CSR mainly as a marketing communication tool. These companies put their resources into CSR activities and CSR-related promotions. However, individual stakeholders' responses are different by what kind of relationship they have with a company. Therefore, targeting is a necessary condition to successful marketing communication strategies. For future research, the effect of multiple identities of individual stakeholders needs to be explained on CSR assessment. 


\subsection{Limitations}

The number of CSR related studies have been increasing explosively in many different fields after the 2000s. Howerev this study reviewed the studies in just management area. To increase understanding CSR, studes in the other fields like sociology, economics, politics, and social welfare need to be reviewed. Additionally, limited studies are review in this study. Much more studies also need to be reviewed including the latest studies.

\section{Reference}

Abbott, W. F., \& Monsen, R. J. (1979). On the Measurement of Corporate Social Responsibility: Self-reported Disclosures as a Method of Measuring Corporate Social Involvement. Academy of Management Journal, 22, 501-515.

Aguilera, R. V., Rupp, D. E., Williams, C. A., \& Ganapathi, J. (2007). Putting the $S$ Back in Corporate Social Responsibility - A Multilevel Theory of Social Change in Organizations. Academy of management Review, 32(3), 836-863.

Ahluwalia, R., Burnkrant, R. E., \& Unnava, H. R. (2000). Consumer Response to Negative Publicity: The Moderating Role of Commitment. Journal of Marketing Research, 37(2), 203-214.

Alexander, G. J., \& Buchholz, R. A. (1978). Corporate Social Responsibility and Stock Market Performance. Academy of Management Journal, 21(3), 479-486.

Anderson, W. T. Jr., \& Cunningham, W. H. (1972). The Socially Conscious Consumer. Journal of Marketing, 36(3), 23-31.

Aupperle, K. E., Carroll, A. B., \& Hatfield, J. D. (1985). An Empirical Examination of the Relationship between Corporate Social Responsibility and Profitability. The Academy of Management Journal, 28(2), 446-463.

Becker-Olsen, K. L., Cudmore, B. A., \& Hill, R. P. (2006). The Impact of Perceived Corporate Social Responsibility on Consumer Behavior. Journal of Business Research, 59(January), 46-53.

Bowen, H. R. (1953). Social Responsibilities of the Businessman. New York, NY: Harper \& Row.

Bowman, E. H., \& Haire, M. (1975). A Strategic Posture toward Corporate Social Responsibility. California Management Review, 18(2), 49-58.

Bragdon, J. H., \& Marlin, J. T. (1972). Is Pollution Profitable? Risk Management, 19(4), 9-18.

Brammer, S., He, H., \& Mellahi, K. (2015). Corporate Social Responsibility, Employee Organizational Identification, and Creative Effort: The Moderating Impact of Corporate Ability. Group and Organization Management, 40, 323-352.

Brown, T. J., \& Dacin, P. A. (1997). The Company and the Product: Corporate Associations and Consumer Product Responses. Journal of Marketing, 61(1), 68-84.

Carroll, A. B. (1991). The Pyramid of Corporate Social Responsibility: Towards the Moral Management of Organizational Stakeholders. Business Horizons,
(July/August), 39-48.

Celma, D., Martínez-Garcia, E., \& Coenders, G. (2014). Corporate Social Responsibility in Human Resource Management: An Analysis of Common Practices and Their Determinants in Spain. Corporate Social Responsibility and Environmental Management, 21, 82-99.

Christensen, C., Craig, T., \& Hart, S. (2001). The Great Disruption. Foreign Affairs, 80(2), 80-96.

Clarkson, M. B. E. (1995). A Stakeholder Framework for Analyzing and Evaluating Corporate Social Performance. Academic Management Review, 20(1), 92-117.

Cochran, P. L., \& Wood, R. A. (1984). Corporate Social Responsibility and Financial Performance. Academy of Management Journal, 27(1), 423-56.

Creyer, E. H., \& Ross, W. T. (1997). The Influence of Firm Behavior on Purchase Intention: Do Consumers Really Care about Business Ethics? Journal of Consumer Marketing, 14(6), 421-432.

David, P., Kline, S., \& Dai, Y. (2005). Corporate Social Responsibility Practices, Corporate Identity, and Purchase Intention: A Dual-Process Model. Journal of Public Relations Research, 17(3), 291-313.

Davis, K., \& Blomstrom, R. L. (1966). Business and its environment. New York, NY: McGraw-Hill.

Day, G. S., \& Montgomery, D. B. (1999). Charting New Directions for Marketing. Journal of Marketing, 63, 3-13.

De Roeck, K., Akremi, A. E., \& Swaen, V. (2016). Consistency Matters! How and When Does Corporate Social Responsibility Affect Employees' Organizational Identification? Journal of Management Studies, 53, 1141-1168.

Roeck, K., Marique, G., Stinglhamber, F., \& Swaen, V. (2014). Understanding Employees' Responses to Corporate Social Responsibility: Mediating Roles of Overall Justice and Organizational Identification. The International Journal of Human Resources Management, 25, 91-112.

Deng, X., \& Xu, Y. (2017). Consumers' Responses to Corporate Social Responsibility Initiatives: The Mediating Role of Consumer-Company Identification. Journal of Business Ethics, 142(3), 515-526.

Donaldson, T., \& Preston, L. E. (1995). The Stakeholder Theory of the Corporation: Concepts, Evidence, and Implications. Academy of Management Review, 20(1), 6591.

Donaldson, T., \& Dunfee, T. W. (1994). Towards a Unified Conception of Business Ethics: Integrative Social Contracts Theory. Academy of Management Review, 19, 252-284.

Donaldson, T., \& Dunfee, T. W. (1999) Ties That Bind: $A$ Social Contracts Approach to Business Ethics. Boston, MA: Harvard Business School Press.

Du, S., Bhattacharya, C. B., \& Sen, S. (2015). Corporate Social Responsibility, Multi-Faceted Job-Products, And Employee Outcomes. Journal of Business Ethics, 131, 319335.

Farooq, M., Farooq, O., \& Jasimuddin, S. M. (2014). Employees Response to Corporate Social Responsibility: Exploring the Role of Employees' Collectivist Orientation. European Management Journal, 32, 916- 927.

Farooq, O., Rupp, D. E., \& Farooq, M. (2017). The Multiple Pathways Through Which Internal and External Corporate Social Responsibility Influence Organizational Identification 
and Multifoci Outcomes: The Moderating Role of Cultural and Social Orientations. Academy of Management Journal, $60,954-985$.

Folkes, V. S., \& Kamins, M. A. (1999). Effects of Information About Firms' Ethical and Unethical Actions on Consumers' Attitudes. Journal of Consumer Psychology, 8(3), 243-259.

Fatima, M., Rahman, Z., \& Khan, I. (2015). Building Company Reputation and Brand Equity Through CSR: The Mediating Role of Trust. International Journal of Bank Marketing, 33(6), 840-856.

Follows, S. B., \& Jobber, D. (2000). Environmentally responsible purchase behaviour: a test of a consumer model. European Journal of Marketing, 34(5/6), 723-746.

Frederick, W. C. (1960). The Growing Concern over Business Responsibility. California Management Review, 2(4), 54-61.

Freeman, R. E. (1997). A Stakeholder Theory of the Corporation. In D. G. Alnord, N. L. Bowie, \& T. L. Beauchamp (eds.). Ethical theory and business. Englewood Cliffs, NJ: Prentice Hall.

Gatignon-Turnau, A. L., \& Mignonac, K. (2015). (Mis)Using Employee Volunteering for Public Relations: Implications for Corporate Volunteers' Organizational Commitment. Journal of Business Research, 68, 7- 18.

Gond, J. P., Akremi, A. E., Swaen, V., \& Babu, N. (2017). The Psychological Microfoundations of Corporate Social Responsibility: A Person-Centric Systematic Review. Journal of Organizational Behavior, 38, 225- 246.

Griffin, J. J., \& Mahon, J. F. (1997). The Corporate Social Performance and Corporate Financial Performance Debate: Twenty-five Years of Incomparable Research. Business and Society, 36(1), 5-31.

Grunig, J. E. (1979). A New Measure of Public Opinions on Corporate Social Responsibility. Academy of Management Journal, 22(4), 738-764.

Guzmán, F., \& Davis, D. (2017). The Impact of Corporate Social Responsibility on Brand Equity: Consumer Responses to Two Types of Fit. Journal of Product and Brand Management, 26(5), 435-446.

Habel, J., Schons, L., Alavi, S., \& Wieseke, J. (2016). Warm Glow or Extra Charge? The Ambivalent Effect of Corporate Social Responsibility Activities on Customers' Perceived Price Fairness. Journal of Marketing, 80, 84-105.

Hart, S. L. (1995). A Natural-Resource-Based View of the Firm. Academy of Management Review, 20(4), 986-1012.

Heald, M. (1988). The Social Responsibilities of Business. Company and Community, 1900-1960. New Brunswick, Canada: Transaction Books.

Hillman, A. J., \& Keim, G. D. (2001). Shareholder Value, Stakeholder Management, and Social Issues: What's the Bottom Line. Strategic Management Journal, 22(2), 125-140.

Ho, F. N., Vitell, S. J., Barnes, J. H., \& Desborde, R. (1997). Ethical Correlates of Role Conflict and Ambiguity in Marketing: The Mediating Role of Cognitive Moral Development. Journal of The Academy of Marketing Science, 25(2), 117-127.

Hur, W., Kim, H., \& Woo, J. (2014). How CSR Leads to Corporate Brand Equity: Mediating Mechanism of Corporate Brand Credibility and Reputation. Journal of Business Ethics, $125,75-86$
Idowu, S. O., Vertigans, S., \& Schiopoiu, A. B. (2017). Corporate Social Responsibility in Times of Crisis. New York, NY: Springer International Publishing.

Joyner, B. E., \& Payne, D. (2002). Evolution and Implementation: A Study of values, Business Ethics and Corporate Social Responsibility. Journal of Business Ethics, 41(4), 297-311.

Kang, C., Germann, F., \& Grewal, R. (2016). Washing Your Sins? Corporate Social Responsibility, Corporate Social Irresponsibility and Firm Performance. Journal of Marketing, $80(2), 59-79$

Karna, J., Hansen, E., \& Juslin, H. (2003). Social Responsibility in Environmental marketing Planning. European Journal of Marketing, 37(5/6), 848-871.

Key, S., \& Popkin, S. J. (1998). Integrating Ethics into the Strategic Management Process: Doing Well by Doing Good. Management Decision, 36(5-6), 331-339.

Kiessling, T., Isaksson, L., \& Yasar, B. (2016). Market Orientation And CSR: Performance Implications. Journal of Business Ethics, 137, 269-284.

Kim, H., Hur, W., \& Yeo, J. (2015). Corporate Brand Trust as Mediator in The Relationship Between Consumer Perception of CSR, Corporate Hypocrisy, And Corporate Reputation. Sustainability, 7, 3683-3694

Kinnear, T. C., Taylor, J. R., \& Ahmed, S. A. (1974) Ecologically Concerned Consumers: Who Are They? Journal of Marketing, 38(April), 20-24.

Klein, J., \& Dawar, N. (2004). Corporate Social Responsibility and Consumers' Attributions and Brand Evaluations in a Product-Harm Crisis. International Journal of Research in Marketing, 21(3), 203-217.

Lee, C., Chang, W., \& Lee, H. (2017). An Investigation of The Effects of Corporate Social Responsibility on Corporate Reputation and Customer Loyalty - Evidence from The Taiwan Non-Life Insurance Industry. Social Responsibility Journal, 2(13), 355-369

Lichtenstein, D. R., Drumwright, M. E., \& Braig, B. M. (2004). The Effect of Corporate Social Responsibility on Customer Donations to Corporate-Supported Nonprofits. Journal of Marketing, 68(4), 16-32.

Litz, R. A. (1996). A Resource-Based-View of the Socially Responsible Firm: Stakeholder Interdependence, Ethical Awareness, and Issue Responsiveness as Strategic Assets. Journal of Business Ethics, 15, 1355-1363.

Liu, M. T., Wong, I. A., Shi, G., Chu, R., \& Brock, J. L. (2014). The Impact of Corporate Social Responsibility (CSR) Performance and Perceived Brand Quality on CustomerBased Brand Preference. Journal of Services Marketing, 28, 181-194.

Luchs, M. G., \& Kumar, M. (2017). "Yes, but this Other One Looks Better/Works Better": How do Consumers Respond to Trade-offs Between Sustainability and Other Valued Attributes? Journal of Business Ethics, 140(3), 567-584.

Luo, X., \& Bhattacharya, C. B. (2006). Corporate Social Responsibility, Customer Satisfaction, and Market Value. Journal of Marketing, 70(October), 1-18.

Luo, X., \& Du, S. (2015). Exploring the Relationship Between Corporate Social Responsibility and Firm Innovation. Marketing Letters, 26, 703-714.

Maignan, I., \& Ferrell, O. C. (2001). Antecedents and Benefits 
of Corporate Citizenship: An Investigation of French Businesses. Journal of Business Research, 51, 37-51.

Maignan, I., \& Ferrell, O. C. (2003). Nature of Corporate Responsibilities Perspectives from American, French, and German Consumers. Journal of Business Research, 56, 55-67.

Marín, L., Cuestas, P. J., \& Román, S. (2016). Determinants of Consumer Attributions of Corporate Social Responsibility. Journal of Business Ethics, 138, 247-260.

Miller, D., \& Merrilees, B. (2013). Linking Retailer Corporate Brand and Environmental Sustainability Practices. Journal of Product and Brand Management, 22(7), 437-443.

Morgeson, F. P., Aguinis, H., Waldman, D. A., \& Siegel, D. S. (2013). Extending Corporate Social Responsibility Research to The Human Resource Management and Organizational Behavior Domains: A Look to The Future. Personnel Psychology, 66, 805-824.

Moskowitz, M. (1972). Choosing Socially Responsible Stocks. Business and Society Review, 1, 71-75.

Narwal, M., \& Singh, R. (2013). Corporate Social Responsibility Practices in India: A Comparative Study of Mncs And Indian Companies. Social Responsibility Journal, 9, 465-478.

Naseer, S., Raja, U., Syed, F., Donai, B. L. M., \& Darr, W. (2016). Perils of Being Close to A Bad Leader in A Bad Environment: Exploring the Combined Effects of Despotic Leadership, Leader Member Exchange, And Perceived Organizational Politics on Behaviors. The Leadership Quarterly, 27, 14-33.

Newman, G. E., Gorlin, M., \& Dhar, R. (2014). When Going Green Backfires: How Firm Intentions Shape the Evaluation of Socially Beneficial Product Enhancements. Journal of Consumer Research, 41, 823-839.

Oberseder, M., Schlegelmilch, B. B., Murphy, P. E., \& Gruber, V. (2014). Consumers' Perceptions of Corporate Social Responsibility: Scale Development and Validation. Journal of Business Ethics, 124(1), 101-115.

Parker, I. R., \& Eilbirt, H. (1975). The Practice of Business Social Responsibility: The Underlying Factors. Business Horizons, 18(4), 5-10.

Perez, A., \& Del Bosque, I. R. (2015). An Integrated Framework to Understand How CSR Affects Customer Loyalty Through Identification, Emotions and Satisfaction. Journal of Business Ethics, 129, 571-584.

Pérez, A., \& Rodríguez-Del-Bosque, I. (2015). Corporate Social Responsibility and Customer Loyalty: Exploring the Role of Identification, Satisfaction and Type of Company. Journal of Services Marketing, 29, 15-25.

Perterson, D. K. (2004). The Relationship Between Perceptions of Corporate Citizenship and Organizational Commitment. Business and Society, 43(3), 296-319.

Porter, M. E., \& Kramer, M. R. (2002). The Competitive Advantage of Corporate Philanthropy. Harvard Business Review, 80(12), 56-69.

Prahalad, C. K. (2002). Strategies for the Bottom of the Economic Pyramid: India as a Source of Innovation. Reflections: The SOL Journal, 3(4), 6-18.

Prahaladand, C. K., \& Hammond, A. (2002). Serving the World's Poor, Profitably. Harvard Business Review, 80(9), 48 $-58$
Roman, R., Hayibor, S., \& Agle, B. R. (1999). The Relationship between Social Performance and Financial Performance. Business and Society, 38(1), 109-125.

Rupp, D. E., Shao, R., Thornton, M., \& Skarlicki, D. P. (2013). Applicants' and Employees' Reactions to Corporate Social Responsibility: The Moderating Effects of First-Party Justice Perceptions and Moral Identity. Personnel Psychology, 66, 895- 993.

Schwepker, C. H., Ferrell, O. C., \& Ingram, T. N. (1997). The Influence of Ethical Climate and Ethical Conflict on Role Stress in the Sales Force. Journal of the Academy of Marketing Science, 25(2), 99-108.

Sen, S., \& Bhattacharya, C. B. (2001). Does Doing Good Always Lead to Doing Better? Consumer Reactions to Corporate Social Responsibility. Journal of Marketing Research, 38(May), 225-243.

Sen, S., Bhattacharya, C. B., \& Korschun, D. (2006). The Role of Corporate Social Responsibility in Strengthening Multiple Stakeholder Relationships: A Field Experiment. Journal of the Academy of Marketing Science, 34(2), pages 158-166.

Sen, S., Du, S., \& Bhattacharya, C. B. (2016). Corporate Social Responsibility: A Consumer Psychology Perspective. Current Opinion in Psychology, 10, 70-75.

Sen, S., Gürhan-Canli, Z., \& Morwitz, V. (2001). Withholding Consumption: A Social Dilemma Perspective on Consumer Boycotts. Journal of Consumer Research, 28(3), 399-417.

Simmon, C. J., \& Becker-Olsen, K. L. (2006). Achieving Marketing Objectives through Social Sponsorships. Journal of Marketing, 70(Oct), 154-169.

Smith, N. C., \& Cooper-Martin, E. (1997). Ethics and Target Marketing: The Role of Product Harm and Consumer Vulnerability. Journal of Marketing, 61(July), 1-20.

Thomas, J. L., Vitell, S. J., Gilbert, F. W., \& Rose, G. M. (2002). The Impact of Ethical Cues on Customer Satisfaction with Service. Journal of Retailing, 78(3), 167-173.

Ullmann, A. A. (1985). Data in Search of a Theory: A Critical Examination of the Relationships among Social Performance, Social Disclosure, and Economic Performance of U. S. Firms. The Academy of Management Review, 10(3), 540-557.

Van Rekom, J., Berens, G., \& Van Halderen, M. (2013). Corporate Social Responsibility: Playing to Win or Playing Not to Lose and Quest; Doing Good by Increasing the Social Benefits of a Company's Core Activities. Journal of Brand Management, 20, 800-814.

Vance, S. (1975). Are Socially Responsible Corporations Good Investment Risks? Management Review, 64(8), 18-24.

Waddock, S. A., \& Graves, S. B. (1997). The Corporate Social Performance-Financial Performance Link. Strategic Management Journal, 18(4), 303-320.

Wartick, S., \& Cochran, P. L. (1985). The Evolution of Corporate Social Performance Model. Academy of Management Review, 10(4), 758-769.

Wernelfelt, B. (1984). A Resource Based View of the Firm. Strategic Management Review, 5, 171-180.

Wood, D. J. (1991). Corporate Social Performance Revisited. Academy of Management Review, 16(4), 691-718. 